\title{
Análisis del ciclo de vida aplicado para la evaluación ambiental en la reutilización del pavimento rígido. Caso de estudio vía Cuenca- Girón- Santa Isabel
}

Life cycle analysis applied for environmental assessment in the reuse of rigid pavement. Case study via Cuenca-Giron-Santa Isabel

Sandy Tamara Orellana Albán. ${ }^{1} \&$ Diego Fernando Coronel Sacoto. ${ }^{2}$

Recibido: 11-08-2021 / Revisado: 22 -08-2021 /Aceptado: 30-08-2021/ Publicado: 05-11-2021

\begin{abstract}
DOI: https://doi.org/10.33262/concienciadigital.v4i4.1.1930

Introduction: The state road network is exposed and vulnerable to natural disasters, and climate change increases the risks of destruction, threatening current and future infrastructure. The geographical location of Ecuador and its geomorphology make the roads of the national network prone to threats of earthquakes, landslides, floods and volcanic activity, conditions that generate interruptions in activities and services that depend on the national road network, added to this, the fact that many roads have already fulfilled their life cycle. Objective: The objective of this research is to analyze the possible environmental impacts that could be reduced with the reuse of rigid pavement, on the Cuenca-Giron-Santa Isabel road, applying the Life Cycle Analysis tool. Methodology: The standardized methodology of Life Cycle Analysis (LCA) was used, as established by ISO 14040, using the SimaPro 9.1.1 software, licensed by the Catholic University of Cuenca, using the cml-ia baseline evaluation method, and the Ecoinvent database, which allows the analysis of impact categories. Results: The results show that the categories with the greatest contribution to environmental impacts are: Abiotic depletion (80\%), Global Warming (40\%), Abiotic depletion (fossil fuels)

\footnotetext{
${ }^{1}$ Universidad Católica de Cuenca, Maestría en Construcciones con Mención en Administración de la Construcción Sustentable. Cuenca, Ecuador. sandy.orellana1@est.ucacue.edu.ec. ORCID https://orcid.org/0000-0002-3008-5557

${ }^{2}$ Universidad Católica de Cuenca, Facultad de Arquitectura, Cuenca, Ecuador. dcoronels@ucacue.edu.ec. ORCID http://orcid.org/0000-0001-8105-4102
} 
(50\%), Human toxicity (80\%), Marine aquatic ecotoxicity (30\%), Freshwater aquatic ecotox $(30 \%)$, and the stage that contributes most to environmental pollution is the stage of transport of heavy cargo with $80 \%$ of emissions this due to the consumption and product of the combustion of diesel and other fuels. Conclusion: The reuse of rigid pavement if it presents less environmental impacts, however, the use and consumption of fuel would determine its feasibility in terms of the costs generated for the reuse of the pavement that has fulfilled its life cycle. The LCA methodology can be used as a tool with certain limitations, for environmental assessment and feasibility for dissemination in the country.

Keywords: life cycle analysis, environmental assessment, environmental impact, pavement reuse.

\section{Resumen}

Introducción: La red vial estatal, está expuesta y es vulnerable a la acción de los desastres naturales, y el cambio climático incrementa los riesgos de destrucción, amenazando la infraestructura actual y futura. La ubicación geográfica del Ecuador y su geomorfología hacen que las carreteras de la red nacional sean propensas a amenazas de terremotos, deslizamientos, inundaciones y actividad volcánica, condiciones que generan interrupciones en actividades y servicios que dependen de la red vial nacional, sumado a esto, el hecho que muchas vías ya han cumplido su ciclo de vida. Objetivo: El objetivo de la presente investigación es analizar los posibles impactos ambientales que se podrían reducir con la reutilización del pavimento rígido, en la vía CuencaGirón- Santa Isabel, aplicando la herramienta de Análisis de Ciclo de Vida. Metodología: Se empleó la metodología estandarizada del Análisis del Ciclo de Vida (ACV), conforme lo establece la Norma ISO 14040, utilizando el software SimaPro 9.1.1, con licencia de la Universidad Católica de Cuenca, utilizando el método de evaluación el CML-IA baseline, y la base de datos Ecoinvent, que permite analizar las categorías de impacto. Resultados: Los resultados muestran que las categorías de mayor contribución a los impactos ambientales son: Agotamiento abiótico (80\%), Calentamiento Global (30\%), Agotamiento abiótico (combustibles fósiles) (50\%), Toxicidad humana (80\%), Ecotoxicidad acuática marina (30\%), Ecotox acuático de agua dulce $(30 \%)$, y la etapa que mayor contribuye a la contaminación ambiental es la etapa de transporte de carga pesada con un $80 \%$ por emisiones esto debido al consumo y producto de la combustión de diésel y otros combustibles. Conclusión: La reutilización del pavimento rígido si presenta menores impactos ambientales, sin embargo, el uso y consumo de combustible determinaría su factibilidad en cuanto a los costos que se generen para la reutilización del pavimento que ha cumplido su ciclo de vida. La metodología ACV puede ser utilizada como herramienta con ciertas limitaciones, para la evaluación ambiental y la factibilidad para su difusión en el país.

Palabras claves: Análisis del ciclo de vida, Evaluación ambiental, Impacto ambiental, Reutilización de Pavimento. 


\section{Introducción}

En América Latina y El Caribe el sector del transporte, en especial la red vial, está expuesta y es vulnerable a la acción de los desastres naturales, y cabe prever que el cambio climático incrementa los riesgos amenazando la infraestructura actual y futura. La infraestructura vial para el transporte es altamente vulnerable a fenómenos climáticos extremos -como tormentas costeras, deslizamientos de tierra, inundaciones y temperaturas extremas-, los cuales pueden deteriorar o incluso destruir la infraestructura vial, ferroviaria, portuaria y aeroportuaria. Los daños causados a los activos de transporte representan buena parte de las pérdidas económicas asociadas a desastres naturales, y la conectividad es un factor esencial para la capacidad de una población y una economía de afrontar y recuperarse de los daños ocasionados por amenazas naturales (Banco Interamericano de Desarrollo [BID], 2018).

Según el BID (2014), las emisiones correspondientes al transporte, el subsector del sector energético que actualmente registra el mayor crecimiento, se duplicaron entre 1980 y 2005, alcanzando un $13 \%$ de las emisiones totales. El transporte vial es la principal fuente de emisiones de $\mathrm{CO} 2$ en el sector de transporte de América Latina y el Caribe, y representa el $93 \%$ de las emisiones totales del sector. De este total, aproximadamente la mitad de las emisiones corresponde al transporte de pasajeros y la otra mitad, al transporte de carga.

La ubicación geográfica del Ecuador y su geomorfología peculiar hacen que las carreteras de la red nacional sean propensas a amenazas de terremotos, deslizamientos, fallas geológicas activas de diferente tipo, inundaciones y actividad volcánica, condiciones que generan interrupciones en actividades y servicios que dependen de la red vial nacional y agravan la situación de otros sectores; como también, incrementan la vulnerabilidad de las comunidades. Entre 2015 y 2019, el Ministerio de Transporte y Obras Públicas invirtió alrededor de USD \$88 millones en reparación de daños y mantenimiento de carreteras relacionados con desastres naturales, siendo el año pico en 2016 después del terremoto con más de USD\$ 37 millones (BID, 2019).

Durante el periodo 2013 - 2017, la industria de la construcción en el Ecuador se ubicó entre los 5 principales sectores que más aportaron al PIB. En este período, el sector de la construcción contribuyó en promedio con el 9.5\% al PIB anualmente (6,584.6 millones de USD), convirtiéndose en el cuarto sector que más contribuye (en promedio) a la economía en el Ecuador (Camino et al., 2018).

En este sentido Baño \& Escalera (2005), afirman que el sector de la construcción contribuye de manera importante a ese deterioro en sus distintas etapas (extracción, transporte, trituradoras, fabricación de materiales, diseño de la edificación y de sus instalaciones que influye decisivamente en el rendimiento energético de la misma, gestión de la obra y de sus residuos...) y necesita dar un giro notable hacia la adopción de decisiones encaminadas hacia la sostenibilidad. 
Por otro lado, la infraestructura de transporte permite el movimiento de bienes, servicios y personas, y por lo tanto es un motor de desarrollo. En efecto, la calidad de la infraestructura está directamente relacionada con el desarrollo económico, la calidad del medio ambiente y la equidad social. Por su parte, la línea base de la sostenibilidad esta cimentada sobre el balance que debe existir entre el desarrollo económico y los impactos sobre el medio ambiente y sobre la sociedad. Por esta razón, la función objetivo de una infraestructura de transporte sostenible puede ser vista como aquella que maximice la calidad de vida de la sociedad y su beneficio económico, además minimice los impactos negativos sobre el ambiente natural (Flintsch \& Bryce, 2014).

El Ministerio de Transporte y Obras Públicas del Ecuador (2013), según la clasificación de carreteras, define que las carreteras sostenibles, deben considerar los aspectos económicos, ecológicos y sociales en el ciclo de vida de la infraestructura de transporte es un prerrequisito para garantizar la movilidad de nuestra sociedad a largo plazo, sostenibilidad seguridad, y eficiencia. La carretera del siglo 21 tiene por objeto establecer un equilibrio entre los aspectos económicos, ecológicos y sociales. Los elementos de la infraestructura vial serán considerados como un todo durante su vida útil, teniendo en cuenta las cuestiones de sostenibilidad en la planificación, elección de materiales y métodos de construcción, mantenimiento y desmontaje, pero, sobre todo la seguridad a todos los usuarios.

Lograr una infraestructura vial sustentable es posible, a través de un diseño de alto desempeño, la reducción de costos y la reducción de impactos ambientales. Se debe equilibrar estos tres factores para presentar una opción sustentable en todos los sentidos, que se pueda llevar a la práctica (MacK et al., 2017).

Un pavimento de larga duración según Mendoza (2014), es aquel en el que no se produce un deterioro significativo en las capas de terracerías y de estructura del mismo, también la capa de rodamiento debe cumplir con un mantenimiento oportuno, para brindar una duración de al menos 35 años.

En este contexto, evaluar la dimensión medioambiental para obtener de los materiales de construcción es intentar calificar y cuantificar el peso de sus impactos durante todo su ciclo de vida, desde la extracción de las materias primas hasta el final del mismo. Por ello es necesario acotar las principales etapas del ciclo de vida de los materiales de construcción tales como: extracción de materias primas o transformación en productos, transporte, construcción, uso, demolición o deconstrucción, y, por último, valorización o depósito de residuos. El bucle se cierra si los residuos son reciclados o los materiales reutilizados (Mercader et al., 2010).

En la presente investigación se plantea la pregunta si ¿la reutilización del pavimento rígido, en el caso de estudio de la vía Cuenca - Girón - Santa Isabel, realizando una evaluación ambiental mediante la aplicación del análisis del ciclo de vida, ¿presenta menores impactos ambientales? 
Para alcanzar las soluciones ambientales necesarias, es indispensable tomar una serie de medidas que permitan cuantificar el impacto que se deja sobre el entorno, y en base a ello tomar decisiones capaces de reducir dichos impactos para ello el objetivo de la investigación es analizar los posibles impactos ambientales que se podrían reducir con la reutilización del pavimento rígido, en la vía Cuenca - Girón - Santa Isabel, aplicando la herramienta de análisis de ciclo de vida.

Una técnica para evaluar los aspectos medioambientales y los potenciales impactos asociados con un producto, proceso o actividad es el Análisis de Ciclo de Vida (ACV). Partiendo de la recolección de un inventario de las entradas y salidas relevantes de un sistema, se evalúan los potenciales impactos medioambientales asociados, para identificar y definir el daño causado a la salud humana y a los sistemas naturales (Rivela, 2012).

Los orígenes en los estudios sobre la consideración del impacto ambiental de un producto/proceso/servicio a lo largo de su ciclo de vida se remontan a la década de 1960, si bien hasta la década de los 90 la metodología de ACV no estuvo suficientemente desarrollada, siendo su aplicación bastante limitada. La complejidad del ACV hacía necesario el establecimiento de un protocolo metodológico estandarizado, que fue elaborado por la International Standard Organization (ISO) y plasmado en la serie ISO 14040 en 1997 (revisada en ISO 14040-44:2006) (Rivela, 2012).

En lo referente a las construcciones de las infraestructuras viales, el estudio realizado por Stripple (2001), adaptó por primera vez, la metodología del ACV para su aplicación en una carretera ubicada en Suecia; en esta investigación se consideran condiciones únicas; tales como, localización, extensión, complejidad de procesos, condiciones meteorológicas, intensidad de tráfico y otros, los cuales se analizan en las etapas de construcción, mantenimiento y tráfico. Los resultados del estudio concluyeron que el mayor impacto ambiental respecto a las emisiones de $\mathrm{CO} 2$, se da en la etapa de tráfico, por la alta circulación de vehículos.

El estudio realizado por Zapata \& Gambatese (2005), compara el pavimento de concreto armado y el pavimento asfáltico, en este estudio se analiza las etapas de extracción de materiales o manufactura, de construcción, operación, de mantenimiento y de fin de vida. Los resultados del estudio concluyeron que el mayor impacto ambiental para el caso del pavimento de concreto armado, se da en la etapa de extracción de materiales o manufactura, debido al mayor consumo de energía (por ende mayor combustión), y para el caso del pavimento asfáltico, se da en la etapa de construcción.

Con respecto al uso del ACV en los países europeos, los cuales tienen políticas más rigurosas con respecto al impacto ambiental, la metodología ACV se cumple de forma eficiente. Puesto que al ser un método flexible, se ajusta a cada proyecto que se vaya a ejecutar, facilitando la implementación de factores sostenibles desde la etapa de diseño en los proyectos viales (Carlson, 2011). 


\section{Metodología}

La presente investigación es de carácter cuantitativa, debido a que buscó cuantificar el impacto ambiental mediante la aplicación de la herramienta de análisis de ciclo de vida en la etapa de fin de uso para la reutilización del pavimento rígido que cumplió su ciclo de vida en el caso de estudio vía Cuenca - Girón - Santa Isabel.

Se utilizó la metodología estandarizada de Análisis de Ciclo de Vida (ACV) conforme lo establece la International Organization for Standardization ([ISO], 2004), que es una herramienta clave para la evaluación de la sostenibilidad y la toma de decisiones de carácter ambiental que contribuye a la elaboración de bases de datos, donde se cuantifique el impacto ambiental como herramienta de apoyo en la toma de decisiones de diseño enfocadas hacia procesos constructivos más sostenibles para la ejecución de infraestructura viales en cuanto a mantenimiento y rehabilitación que garantice su buen estado y disminuyan los costos.

\section{Marco Teórico}

Dentro de las normas INEN-ISO 14040 se trata el tema específico del ACV. Esta norma indica que un estudio de $\mathrm{ACV}$, como se observa en la figura 1, consta de cuatro fases:

Figura 1

Fases de un Análisis de Ciclo de Vida

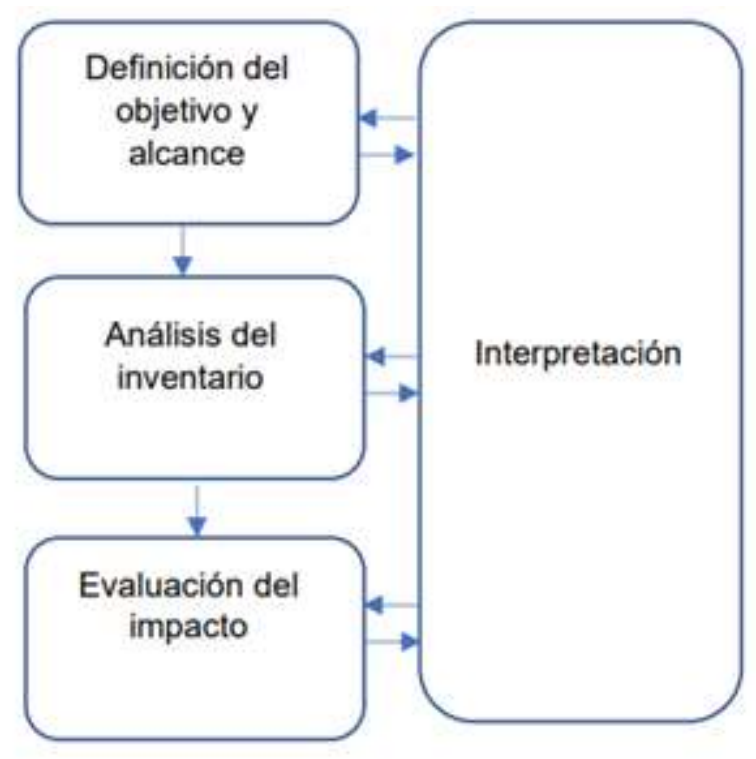

Fuente: Basado en la Norma INEN-ISO 14040 (2004)

En Ecuador se adoptaron estas normas que según la investigación realizada por Remache-Vinueza (2017), se alinean con lo establecido en la codificación de la Ley de Gestión Ambiental en su Capítulo II DE LA EVALUACIÓN DE IMPACTO AMBIENTAL Y DEL CONTROL AMBIENTAL, que establece que toda institución sea de carácter pública o privada, que se encuentre desarrollando un proyecto que 
suponga un riesgo ambiental, tiene la obligación de previo a su ejecución, sobrellevar un proceso de evaluación de impacto ambiental.

Para el autor Perelli \& Ruiz (2017), una aproximación del análisis del ciclo de vida $(\mathrm{ACV})$ al caso particular de carretera, tiene las siguientes definiciones:

a) Definición del objetivo, y alcance del sistema. Esta primera fase es fundamental ya que es la que constituye el marco en el que se va a realizar el estudio. Se debe definir el objetivo del estudio, el alcance, de acuerdo con los límites del sistema.

Límites del sistema: Se establecen los límites del sistema ya que implica definir los procesos que se van a incluir en el sistema, en función de los objetivos del estudio.

En las Fases del ciclo de vida de una carretera hay un elevado número de elementos a considerar, como se puede observar en la Figura 2, por lo que se deberá considerar hacerlo de forma sólo parcial, no considerando todas las fases (Perelli \& Ruiz, 2017).

Figura 2

Fases del ciclo de vida de una carretera habitualmente consideradas

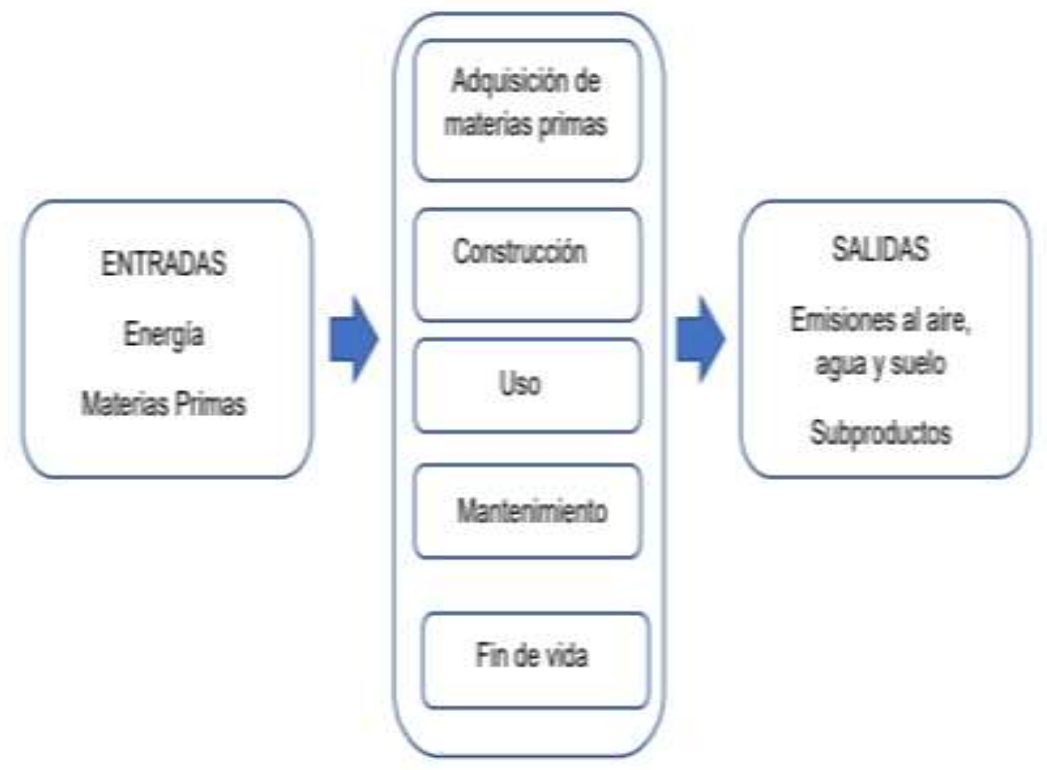

Fuente: Basado en Perelli \& Ruiz (2017)

Unidad Funcional (UF). Es la medida relevante del sistema y a la que irán referidos todos los datos del sistema.

Las unidades funcionales según García-Navarro et al. (2012), deberán considerar dos aspectos importantes:

- Que haya un método fiable para medir la UF seleccionada.

- Que se garantice la equivalencia de funciones para que puedan utilizarse en las comparaciones. 
Análisis de inventario de Ciclo de Vida (ICV). Consiste en la elaboración de un inventario de los datos de entrada y salida en el sistema estudiado. En esta etapa se recogen los datos correspondientes a las entradas y salidas para todos los procesos del sistema: las materias primas, el agua, la energía, las emisiones, los vertidos y residuos, etc.

Evaluación de impacto del Ciclo de Vida (EICV). Esta tercera etapa (EICV), que consiste en traducir los resultados de la etapa anterior (ICV) a potenciales impactos medioambientales.

Según Perelli \& Ruiz (2017), en esta etapa hay que distinguir tres aspectos fundamentales:

1. Las categorías de impacto: representan los impactos ambientales que se consideran de interés; es decir, aquellos de los cuales se desean obtener resultados. Existe multitud de categorías de impacto ambiental y la selección de unas u otras dependerá del objetivo, del destinatario y del nivel de exactitud de los resultados requeridos. En la tabla 1, se indican las principales categorías de impacto ambiental contempladas por la SETAC.

2. El Indicador de categoría o Ecoindicador: es la medida cuantitativa o unidad de referencia establecida para cada categoría de impacto ambiental (por ejemplo, $\mathrm{kg}$ CO2eq para la categoría de Calentamiento Global) a la que representa.

3. El modelo de caracterización: describe la metodología utilizada para convertir los datos del ICV en indicadores de categoría.

Tabla 1

Principales categorías de impacto ambiental contempladas por la SETAC

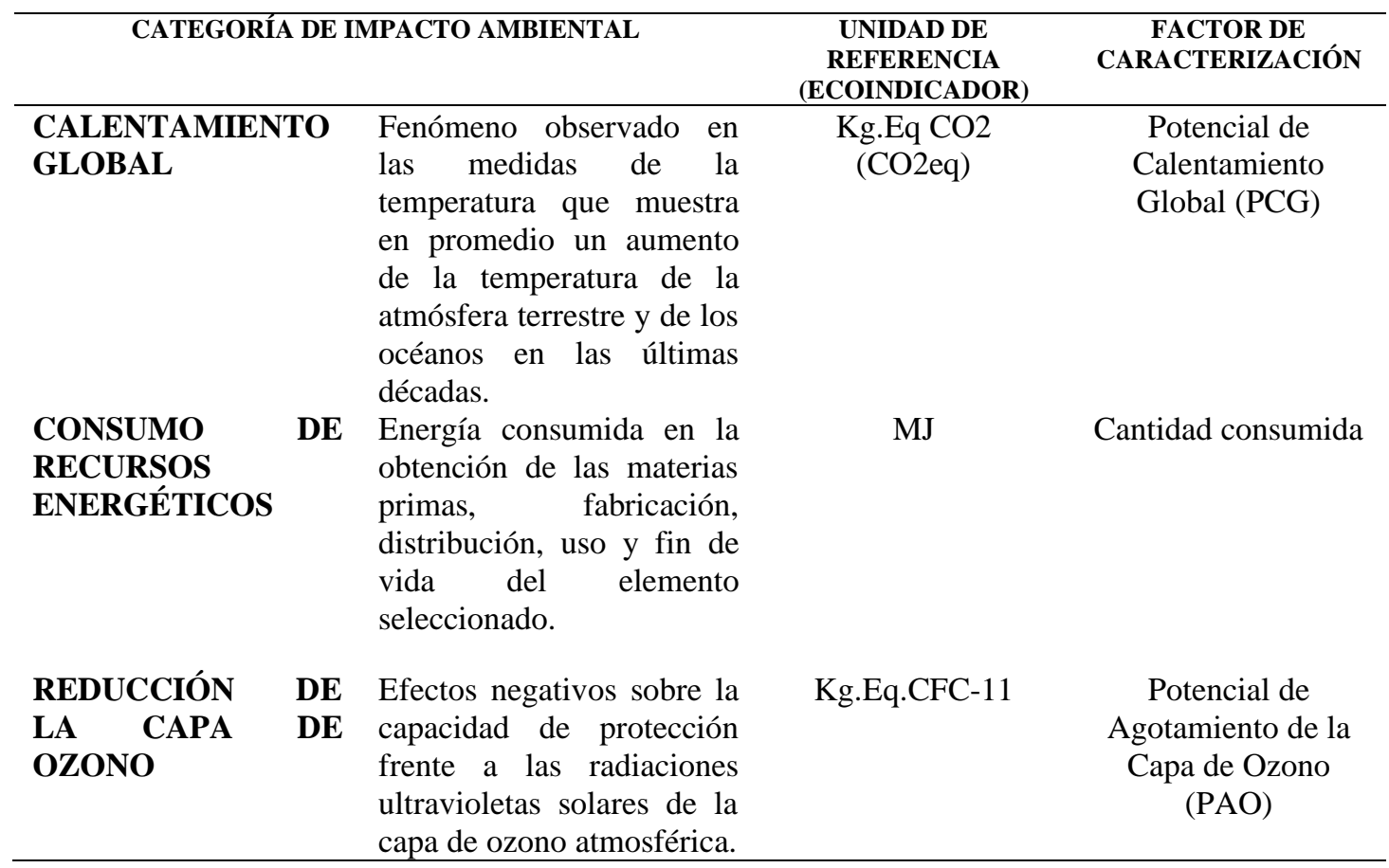


Tabla 1

Principales categorías de impacto ambiental contempladas por la SETAC (continuación)

\begin{tabular}{|c|c|c|c|c|}
\hline \multicolumn{3}{|c|}{ CATEGORÍA DE IMPACTO AMBIENTAL } & \multirow{2}{*}{$\begin{array}{c}\begin{array}{c}\text { UNIDAD DE REFERENCIA } \\
\text { (ECOINDICADOR) }\end{array} \\
\mathrm{Kg} . \mathrm{Eq} . \mathrm{NO}_{2}\end{array}$} & \multirow{2}{*}{$\begin{array}{c}\text { FACTOR DE } \\
\text { CARACTERIZACIÓN } \\
\text { Potencial de Eutrofización (PE) }\end{array}$} \\
\hline EUTROFIZACIÓN & & $\begin{array}{l}\text { Crecimiento excesivo de la } \\
\text { población de algas originado } \\
\text { por el enriquecimiento } \\
\text { artificial de las aguas de ríos } \\
\text { y embalses como } \\
\text { consecuencia del empleo } \\
\text { masivo de fertilizantes y } \\
\text { detergentes que provoca un } \\
\text { alto consumo del oxígeno } \\
\text { del agua. }\end{array}$ & & \\
\hline $\begin{array}{l}\text { CALENTAMIENTO } \\
\text { GLOBAL }\end{array}$ & & $\begin{array}{l}\text { Fenómeno observado en las } \\
\text { medidas de la temperatura } \\
\text { que muestra en promedio un } \\
\text { aumento de la temperatura } \\
\text { de la atmósfera terrestre y de } \\
\text { los océanos en las últimas } \\
\text { décadas. }\end{array}$ & $\begin{array}{l}\mathrm{Kg} . \mathrm{Eq} \mathrm{CO} 2 \\
(\mathrm{CO} 2 \mathrm{eq})\end{array}$ & $\begin{array}{l}\text { Potencial de Calentamiento } \\
\text { Global (PCG) }\end{array}$ \\
\hline $\begin{array}{l}\text { CONSUMO } \\
\text { RECURSOS } \\
\text { ENERGÉTICOS }\end{array}$ & DE & $\begin{array}{l}\text { Energía consumida en la } \\
\text { obtención de las materias } \\
\text { primas, fabricación, } \\
\text { distribución, uso y fin de } \\
\text { vida del elemento } \\
\text { seleccionado. }\end{array}$ & MJ & Cantidad consumida \\
\hline $\begin{array}{l}\text { REDUCCIÓN DE } \\
\text { CAPA DE OZONO }\end{array}$ & LA & $\begin{array}{l}\text { Efectos negativos sobre la } \\
\text { capacidad de protección } \\
\text { frente a las radiaciones } \\
\text { ultravioletas solares de la } \\
\text { capa de ozono atmosférica. }\end{array}$ & Kg.Eq.CFC-11 & $\begin{array}{l}\text { Potencial de Agotamiento de la } \\
\text { Capa de Ozono (PAO) }\end{array}$ \\
\hline ACIDIFICACIÓN & & $\begin{array}{l}\text { Pérdida de la capacidad } \\
\text { neutralizante del suelo y del } \\
\text { agua, como consecuencia del } \\
\text { retorno a la superficie de la } \\
\text { tierra, en forma de ácidos de } \\
\text { los óxidos de azufre y } \\
\text { nitrógeno descargados a la } \\
\text { atmósfera. }\end{array}$ & Kg. Eq SO$~_{2}$ & Potencial de Acidificación (PA) \\
\hline $\begin{array}{l}\text { CONSUMO } \\
\text { MATERIAS PRIMAS }\end{array}$ & DE & $\begin{array}{l}\text { Consumo de materiales } \\
\text { extraídos de la naturaleza. }\end{array}$ & TM & Cantidad Consumida \\
\hline $\begin{array}{l}\text { FORMACIÓN } \\
\text { OXIDANTES } \\
\text { FOTOQUÍMICOS }\end{array}$ & DE & $\begin{array}{l}\text { Formación de los } \\
\text { precursores que dan lugar a } \\
\text { la contaminación } \\
\text { fotoquímica. La luz solar } \\
\text { incide sobre dichos } \\
\text { precursores, provocando la } \\
\text { formación de una serie de } \\
\text { compuestos conocidos como } \\
\text { oxidantes fotoquímicos (el } \\
\text { ozono-O }{ }_{3} \text { es el más } \\
\text { importante por su } \\
\text { abundancia y toxicidad). }\end{array}$ & Kg. Eq. $\mathrm{C}_{2} \mathrm{H}_{2}$ & $\begin{array}{l}\text { Potencial de Formación de } \\
\text { Oxidantes Fotoquímicos } \\
\text { (PFOF) }\end{array}$ \\
\hline
\end{tabular}

Fuente: Perelli \& Ruiz (2017) 
Selección de categorías de impacto, indicadores de categoría y modelos de caracterización

Consiste en traducir los resultados de la etapa Inventario de Ciclo de Vida a potenciales impactos medioambientales.

Clasificación: Asignación de los resultados del Inventario de Ciclo de Vida a las distintas categorías de impacto según el modelo de caracterización asignado.

Caracterización: Se denomina al cálculo de los resultados de cada categoría de impacto. Los datos del ICV se clasifican en categorías de impacto ambiental de acuerdo con el efecto de cada una sobre el ambiente.

Normalización: Vinculando los resultados a una unidad de referencia (zona geográfica, etc.).

Interpretación del ciclo de vida. En esta fase se extraen todos los resultados derivados de las dos etapas anteriores, en la línea de los objetivos y alcance del estudio, y que puedan proporcionar conclusiones y recomendaciones para la toma de decisiones en lo relativo a estrategia de producto.

En este contexto, la presente investigación se justifica ya que en el Ecuador, se dispone de poca información respecto al uso de esta herramienta en la construcción de las carreteras y autopistas, que permitan realizar una evaluación ambiental para disminuir los impactos ambientales.

\section{Caso de estudio: vía Cuenca- Girón- Santa Isabel}

La vía Cuenca - Girón - Pasaje, especialmente el tramo entre Girón y Santa Isabel (figura 3), presenta problemas geotécnicos en su calzada producto de varios fenómenos geológicos que no se pueden controlar como fallas, deslizamientos, flujos, agrietamientos de suelo, etc., Este problema es constante y a pesar de los arreglos realizados en la vía los daños siguen latentes (Ávila, 2020), y empeoran con el transcurso del tiempo.

De acuerdo al plan de desarrollo y ordenamiento territorial del Azuay actualizado 2015 - 2030 del Gobierno Provincial de Azuay (2018), la red vial estatal que se emplaza en la Provincia del Azuay ha sido intervenida en los últimos años, encontrándose en buen estado un porcentaje similar al $74 \%$ en tanto que el restante $26 \%$, se encuentran en mal estado, estas son las vías, Léntag- San Francisco-Uzhcurrumi (límite con la provincia de El Oro) y Sevilla de Oro - La Sopladora, y Gualaceo - La Virgen.

La red vial de la provincia del Azuay según el Gobierno Provincial de Azuay (2018), tiene una longitud vial aproximada de $4.121 \mathrm{~km}$, de éste valor el 14\% corresponde a la red Estatal que representan aproximadamente $565 \mathrm{Km}$; de los restantes $3556 \mathrm{~km}$, es decir el $86 \%$ de la red vial de la provincia, el $6 \%$ corresponde a la red secundaria que 
representan $237 \mathrm{Km}$; en tanto que la red terciaria representa el $26 \%$ con $1055 \mathrm{Km}$, dejando para la red vecinal el $55 \%$ de la Red con 2.264 Km de vías.

Figura 3

Fases del ciclo de vida de una carretera habitualmente consideradas

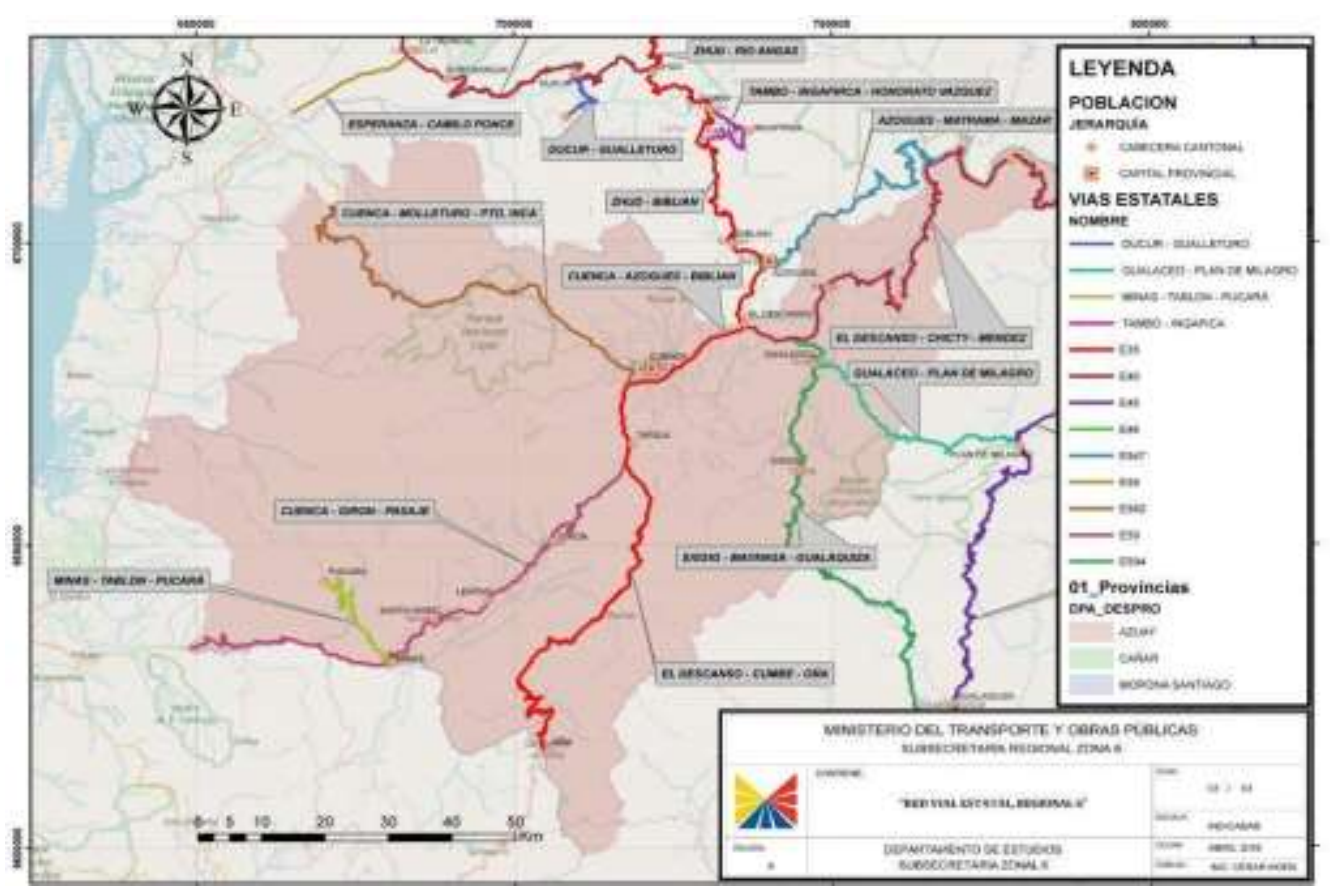

Fuente: Ministerio del Transporte y Obras Públicas

\section{Resultados}

\section{Definición del objetivo, y alcance del sistema}

El objetivo de este estudio fue cuantificar los posibles impactos ambientales a través de la obtención de los valores de categorías de impacto del pavimento rígido que ha cumplido su ciclo de vida, aplicando la herramienta de Análisis de Ciclo de Vida (ACV) para ser reutilizado en la reconstrucción de la vía Cuenca- Girón- Santa Isabel que forma parte de la Red vial estatal. Se encuentra ubicada al Sur del Ecuador, Suroeste de la Provincia del Azuay, a tan sólo $62 \mathrm{Km}$. de la Ciudad de Cuenca, siguiendo la carretera Cuenca - Girón - Pasaje.

Para el caso de estudio, la unidad funcional se definió: un $\mathrm{m}^{3}$ de hormigón obtenido a partir del pavimento que ha cumplido su ciclo de vida, incluyéndose dentro del alcance la fase fin o final de vida (de la cuna a la cuna o cradle to cradle).

Límites del sistema: Según el autor Perelli \& Ruiz (2017), para la fase de fin de vida dependiendo de los límites del sistema que se establezcan, esta fase puede incluir la demolición, el depósito en vertedero, los procesos de reciclaje así como otras actividades que tengan lugar tras la retirada del firme.

Para el caso de estudio se han establecido los límites de acuerdo al esquema de la figura 4 , ya que el objetivo de la investigación fue analizar los posibles impactos ambientales 
que se podrían reducir con la reutilización del pavimento rígido, en la vía Cuenca Girón - Santa Isabel, aplicando la herramienta de análisis de ciclo de vida para ello se han agrupado en tres etapas: demolición, transporte y reutilización que agrupan los procesos que han sido evaluados en función de los objetivos.

\section{Figura 4}

Límites del sistema para fase de fin de vida del pavimento

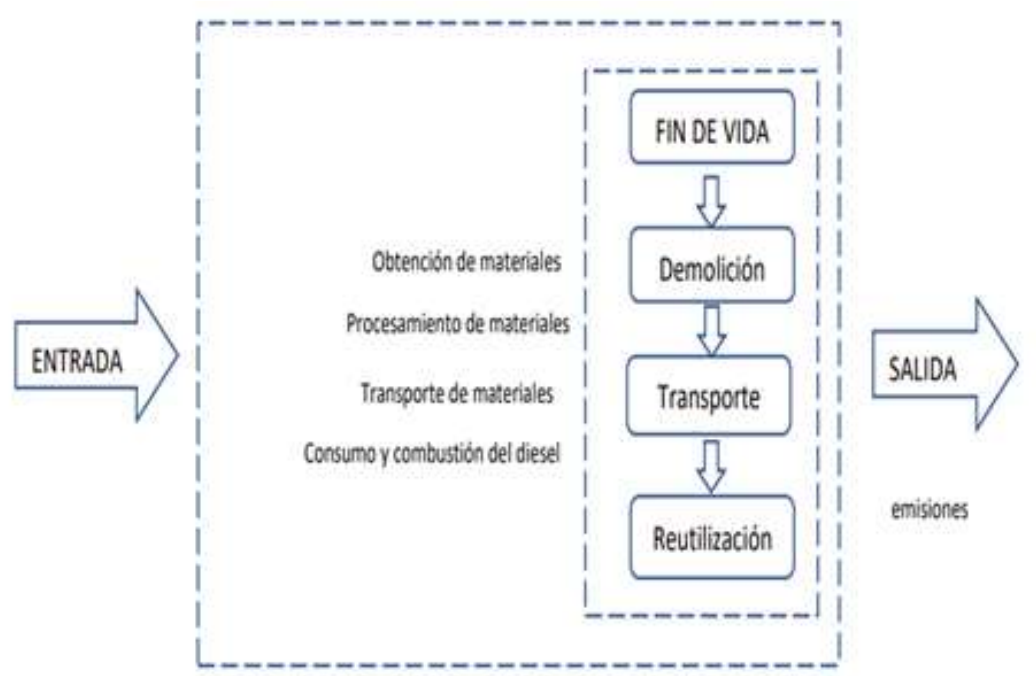

Análisis de inventario de Ciclo de Vida (ICV)

En esta fase se han definido los procesos unitarios que se evaluaron para la obtención de un $\mathrm{m}^{3}$ de hormigón que cumplió su ciclo de vida, mismas que se han agrupado en tres etapas que son demolición, transporte, y reutilización. En la tabla 2 se detallan las variables evaluadas, que se relacionaron con la unidad funcional.

\section{Tabla 2}

Etapas del proceso y procesos unitarios consideradas en el fin de vida del pavimento

\begin{tabular}{|c|c|c|c|c|}
\hline Etapas & Proceso unitario & Nomb & e en base de datos en & imapro \\
\hline Demolición & $\begin{array}{l}\mathrm{M}^{3} \text { de hormigón } \\
\text { obtenido por } \\
\text { trituración de } \\
\text { hormigón que } \\
\text { cumplio CV, } \\
\text { representado como } \\
\text { consumo y } \\
\text { combustión del } \\
\text { diésel, que se } \\
\text { requiere para } \\
\text { transporte en } \\
\text { camiones pesados. }\end{array}$ & $\begin{array}{l}\text { Diesel }\{\mathrm{GLO}\} \mid \\
\text { market group for | } \\
\text { Cut-off, U }\end{array}$ & $\begin{array}{l}\text { Excavation, } \\
\text { hydraulic digger } \\
\{\text { GLO }\} \mid \text { market for } \mid \\
\text { Cut-off, } U\end{array}$ & $\begin{array}{l}\text { Transport, freight, } \\
\text { lorry } 16-32 \text { metric } \\
\text { ton, euro3 }\{\text { RoW }\} \mid \\
\text { market for } \\
\text { transport, freight, } \\
\text { lorry 16-32 metric } \\
\text { ton, }\end{array}$ \\
\hline Transporte & $\begin{array}{l}\text { M3 de hormigón } \\
\text { obtenido por } \\
\text { transporte de arena } \\
\text { y grava transportado } \\
\text { desde la mina, } \\
\text { representado como } \\
\text { consumo y } \\
\text { combustión del } \\
\text { diésel. }\end{array}$ & $\begin{array}{l}\text { Diesel }\{\mathrm{GLO}\} \mid \\
\text { market group for | } \\
\text { Cut-off, } \mathrm{U}\end{array}$ & $\begin{array}{l}\text { Transport, freight, } \\
\text { lorry 16-32 metric } \\
\text { ton, euro3 \{RoW\}| } \\
\text { market for } \\
\text { transport, freight, } \\
\text { lorry 16-32 metric } \\
\text { ton, EURO3 | Cut- } \\
\text { off, U }\end{array}$ & \\
\hline
\end{tabular}




\section{Tabla 2}

Etapas del proceso y procesos unitarios consideradas en el fin de vida del pavimento(continuación)

\begin{tabular}{|c|c|c|c|c|c|}
\hline Etapas & Proceso unitario & Nombr & n base de datos en & napro & \\
\hline Demolición & $\begin{array}{lr}\mathrm{M}^{3} \quad \text { de } & \text { hormigón } \\
\text { obtenido } & \text { por } \\
\text { trituración } & \text { de } \\
\text { hormigón } & \text { que } \\
\text { cumplio } & \mathrm{CV}, \\
\text { representado } & \text { como } \\
\text { consumo } & \mathrm{y} \\
\text { combustión } & \text { del } \\
\text { diésel, que } & \text { se } \\
\text { requiere } & \text { para } \\
\text { transporte } & \text { en } \\
\text { camiones pesados. }\end{array}$ & $\begin{array}{c}\text { Diesel }\{\mathrm{GLO}\} \mid \\
\text { market group for } \mid \\
\text { Cut-off, } \mathrm{U}\end{array}$ & $\begin{array}{c}\text { Excavation, } \\
\text { hydraulic digger } \\
\{\text { GLO }\} \mid \text { market for } \\
\mid \text { Cut-off, } U\end{array}$ & $\begin{array}{c}\text { Transport, } \\
\text { freight, lorry } 16- \\
32 \text { metric ton, } \\
\text { euro3 }\{\text { RoW }\} \mid \\
\text { market for } \\
\text { transport, freight, } \\
\text { lorry } 16-32 \\
\text { metric ton, }\end{array}$ & \\
\hline Transporte & $\begin{array}{lr}\text { M3 de hormigón } \\
\text { obtenido } & \text { por } \\
\text { transporte de } & \text { arena } \\
\text { y grava transportado } \\
\text { desde la mina, } \\
\text { representado como } \\
\text { consumo } \\
\text { combustión } \\
\text { diésel. }\end{array}$ & $\begin{array}{c}\text { Diesel }\{\text { GLO }\} \mid \\
\text { market group for } \mid \\
\text { Cut-off, } U\end{array}$ & $\begin{array}{l}\text { Transport, freight, } \\
\text { lorry } 16-32 \text { metric } \\
\text { ton, euro3 }\{\text { RoW }\} \mid \\
\text { market for } \\
\text { transport, freight, } \\
\text { lorry } 16-32 \text { metric } \\
\text { ton, EURO3 | Cut- } \\
\text { off, } U\end{array}$ & & \\
\hline Reutilización & $\begin{array}{l}\text { Por otra parte, para } \\
\text { un metro cubico de } \\
\text { hormigón se } \\
\text { requiere una hora de } \\
\text { trituradora, y en la } \\
\text { mina se requiere } \\
\text { excavadora para } \\
\text { sacar material del } \\
\text { río, volquete para } \\
\text { conducir el material } \\
\text { a tamizadora para } \\
\text { separar arena grava } \\
\text { y piedra, y luego se } \\
\text { requiere cargadora } \\
\text { para cargar los } \\
\text { áridos a los } \\
\text { volquetes. }\end{array}$ & $\begin{array}{l}\text { Diesel }\{\text { GLO }\} \mid \\
\text { market group for } \mid \\
\text { Cut-off, } \mathrm{U}\end{array}$ & $\begin{array}{l}\text { Transport, freight, } \\
\text { lorry } 16-32 \text { metric } \\
\text { ton, euro3 }\{\text { RoW }\} \mid \\
\text { market for } \\
\text { transport, freight, } \\
\text { lorry } 16-32 \text { metric } \\
\text { ton, EURO3 | Cut- } \\
\text { off, } U\end{array}$ & $\begin{array}{c}\text { Excavation, } \\
\text { hydraulic digger } \\
\{\text { GLO }\} \mid \text { market } \\
\text { for } \mid \text { Cut-off, U }\end{array}$ & $\begin{array}{l}\text { Transport, } \\
\text { freight, } \\
\text { conveyor } \\
\text { belt }\{\mathrm{GLO}\} \mid \\
\text { market for } \\
\text { transport, } \\
\text { freight, } \\
\text { conveyor } \\
\text { belt } \mid \text { Cut- } \\
\text { off, } \mathrm{U}\end{array}$ \\
\hline
\end{tabular}

Fuente: En base a Simapro (PRÉ CONSULTANTS BV, 2018).

Nota: La elección de los materiales se ha llevado a cabo según el sistema de abreviación que representa la localización geográfica (Ecoinvent.org, s.f.). Se ha elegido RoW (Rest-of-theWorld) o GLO (Global), ya que ambas abreviaciones significan lo mismo.

\section{Evaluación de impacto del Ciclo de Vida (EICV)}

Para la evaluación del impacto, se utilizó el software SimaPro 9.1.1, con licencia de la Universidad Católica de Cuenca, usando el método de evaluación el CML-IA baseline, y la base de datos Ecoinvent.

SimaPro es un programa desarrollado por la empresa holandesa PRéConsultants, que permite realizar ACV mediante el uso de bases de datos de inventario propias (creadas por el usuario) y bibliográficas (Ecoinvent, BUWAL, IDEMAT, ETH, IVAM). 
Con esta herramienta se facilita el análisis y la representación gráfica de ciclos complejos de un modo sistemático y transparente. El programa ayuda a aplicar eficazmente su experiencia en ACV, para ayudarlo a potenciar la toma de decisiones sólida, mejorar los ciclos de vida de sus productos y mejorar el impacto positivo de su empresa. SimaPro es el paquete de software ACV líder, con una reputación de 25 años en la industria y la academia en más de 80 países (PRÉ CONSULTANTS BV, 2018).

Caracterización. En la tabla 3, se muestra los resultados de la caracterización de las etapas de demolición, transporte, y reutilización a partir de datos asignados a cada categoría de impacto, que arrojan valores positivos es decir perjudiciales para el ambiente.

Los valores resultantes del cálculo en el Simapro se exportaron a Excel. La información obtenida se procesó a través de tablas y cuadros comparativos para su análisis y sus correspondientes gráficas.

Se aprecia en la figura 5, los resultados obtenidos del software Simapro para la caracterización se observa que se encuentran con mayores contribuciones las categorías de impacto dadas por: agotamiento abiótico (combustibles fósiles), calentamiento global, toxicidad humana, ecotoxicidad acuática marina, ecotox acuático de agua dulce, y en menores porcentajes para las categorías de impacto: de agotamiento abiótico, agotamiento de la capa de ozono (PAO), ecotoxicidad terrestre, oxidación fotoquímica, acidificación, y eutrofización.

La mayoría de impactos ambientales se producen por el consumo y combustión de diésel debido a las emisiones consecuencia del uso de maquinaría y el transporte de carga pesada que generan el mayor porcentaje de impactos ambientales, contribuyendo mayormente en el calentamiento global, agotamiento abiótico (combustibles fósiles), y en toxicidad humana, en las tres etapas de demolición, transporte y reutilización.

Tabla 3

Resultados de la caracterización para categorías de impacto

\begin{tabular}{|c|c|c|c|c|}
\hline $\begin{array}{l}\text { Categoría de } \\
\text { impacto }\end{array}$ & Unidad & Demolición & Transporte & Reutilización \\
\hline Agotamiento abiótico & $\mathrm{kg} \mathrm{Sb}$ eq & $4.14 \mathrm{E}+02$ & $4.05 \mathrm{E}+02$ & $4.14 \mathrm{E}+02$ \\
\hline $\begin{array}{l}\text { Agotamiento abiótico } \\
\text { (combustibles } \\
\text { fósiles) }\end{array}$ & MJ & $75,598,045$ & $74,874,749$ & $75,598,044$ \\
\hline $\begin{array}{l}\text { Calentamiento global } \\
\text { (GWP100a) }\end{array}$ & $\mathrm{kg} \mathrm{CO} 2 \mathrm{eq}$ & $77,273,111$ & $72,004,296$ & $77,273,111$ \\
\hline $\begin{array}{l}\text { Agotamiento de la } \\
\text { capa de ozono (ODP) }\end{array}$ & kg CFC-11 eq & $9.72 \mathrm{E}+01$ & $9.63 \mathrm{E}+01$ & $9.72 \mathrm{E}+01$ \\
\hline
\end{tabular}




\section{Tabla 3}

Resultados de la caracterización para categorías de impacto (continuación)

\begin{tabular}{|c|c|c|c|c|}
\hline $\begin{array}{l}\text { Categoría de } \\
\text { impacto }\end{array}$ & Unidad & Demolición & Transporte & Reutilización \\
\hline Toxicidad humana & $\mathrm{kg} \mathrm{1,4-DB} \mathrm{eq}$ & $36,146,294$ & $34,759,657$ & $36,146,294$ \\
\hline $\begin{array}{l}\text { Ecotox acuático de } \\
\text { agua dulce. }\end{array}$ & $\mathrm{kg} 1,4-\mathrm{DB}$ eq & $13,700,617$ & $13,079,961$ & $13,700,617$ \\
\hline $\begin{array}{l}\text { Ecotoxicidad } \\
\text { acuática marina }\end{array}$ & $\mathrm{kg} 1,4-\mathrm{DB}$ eq & $37,702,047$ & $36,559,027$ & $37,702,047$ \\
\hline $\begin{array}{l}\text { Ecotoxicidad } \\
\text { terrestre }\end{array}$ & $\mathrm{kg} \mathrm{1,4-DB} \mathrm{eq}$ & 0,015077613 & 0,014590593 & 0,015077613 \\
\hline $\begin{array}{l}\text { Oxidación } \\
\text { fotoquímica }\end{array}$ & $\mathrm{kg} \mathrm{C} 2 \mathrm{H} 4 \mathrm{eq}$ & 0,0031972069 & 0,0031024708 & 0,0031972069 \\
\hline Acidificación & $\mathrm{kg} \mathrm{SO} 2 \mathrm{eq}$ & 0,080164409 & 0,076265182 & 0,080164409 \\
\hline Eutrofización & kg PO4--- eq & 0,010622129 & 0,0096874433 & 0,010622129 \\
\hline
\end{tabular}

Fuente: Elaboración propia a partir de datos de Simapro (PRÉ CONSULTANTS BV, 2018)

\section{Figura 5}

Resultados de la Caracterización para Categorías de Impacto

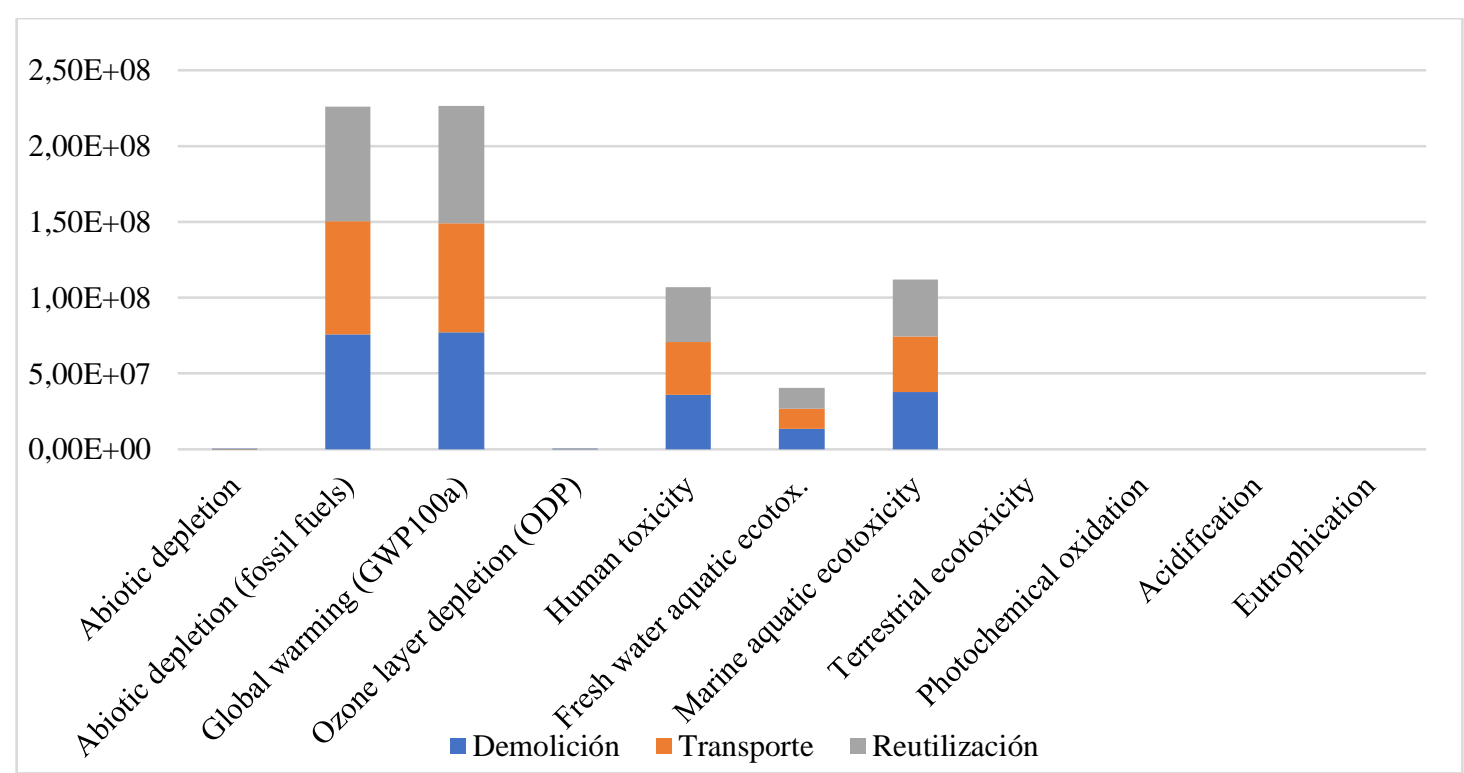

Fuente: Elaboración propia a partir de datos de SimaPro (PRÉ CONSULTANTS BV, 2018)

Normalización. En la tabla 4 se muestran los resultados obtenidos para todas las categorías de impacto, a través de la normalización en las tres etapas que se agruparon los procesos para demolición, transporte y reutilización. 
En la Figura 6, se puede observar que las categorías de impactos con mayor contribución son: Agotamiento abiótico (80\%), agotamiento abiótico (combustibles fósiles) (50\%), calentamiento global (30\%), toxicidad humana (80\%), ecotoxicidad acuática marina (30\%), ecotox acuático de agua dulce (30\%).

Para la etapa de demolición se han obtenido como el mayor impacto ambiental al indicador de categoría de agotamiento abiótico (combustibles fósiles) con un valor del $50 \%$.

En la etapa de transporte la mayor contribución corresponde a la categoría de agotamiento abiótico $(80 \%)$ y toxicidad humana (80\%), en menor contribución con un valor de $30 \%$ el calentamiento global.

En cuanto a la etapa de reutilización se la categoría de impacto con mayor valor corresponde al agotamiento abiótico (combustibles fósiles) (MJI) un 50\%.

Por lo que estos resultados nos indican que en la etapa de transporte se generan los mayores impactos ambientales con una contribución de más del $80 \%$, esto debido al uso de combustibles en el transporte de los materiales y durante la utilización de maquinaria, así como también los vertidos de líquidos al suelo y agua producto del diésel y combustible, y por el uso de maquinaría para la trituración y obtención de hormigón a partir de pavimento que ha cumplido su ciclo de vida.

\section{Tabla 4}

Resultados de la normalización para categorías de impacto

\begin{tabular}{|c|c|c|c|c|}
\hline $\begin{array}{l}\text { Categoría } \\
\text { impacto }\end{array}$ & Unidad & Demolición & Transporte & Reutilización \\
\hline $\begin{array}{l}\text { Agotamiento } \\
\text { abiótico }\end{array}$ & $\mathrm{kg} \mathrm{Sb} \mathrm{eq}$ & $4.89 \mathrm{E}-07$ & $4.78 \mathrm{E}-06$ & $4.89 \mathrm{E}-07$ \\
\hline $\begin{array}{l}\text { Agotamiento } \\
\text { abiótico } \\
\text { (combustibles } \\
\text { fósiles) }\end{array}$ & MJ & $2.40 \mathrm{E}-04$ & $2.38 \mathrm{E}-05$ & $2.40 \mathrm{E}-04$ \\
\hline $\begin{array}{l}\text { Calentamiento } \\
\text { global (GWP100a) }\end{array}$ & $\mathrm{kg} \mathrm{CO} 2 \mathrm{eq}$ & $1.54 \mathrm{E}-05$ & $1.43 \mathrm{E}-05$ & $1.54 \mathrm{E}-05$ \\
\hline $\begin{array}{l}\text { Agotamiento de la } \\
\text { capa de ozono } \\
\text { (ODP) }\end{array}$ & $\mathrm{kg} \mathrm{CFC}-11 \mathrm{eq}$ & $1.09 \mathrm{E}-06$ & $1.08 \mathrm{E}-06$ & $1.09 \mathrm{E}-06$ \\
\hline Toxicidad humana & $\mathrm{kg} 1,4-\mathrm{DB}$ eq & 4.66E-07 & 4.48E-06 & 4.66E-07 \\
\hline $\begin{array}{l}\text { Ecotox acuático de } \\
\text { agua dulce. }\end{array}$ & $\mathrm{kg}$ 1,4-DB eq & 2.64E-05 & $2.52 \mathrm{E}-05$ & 2.64E-05 \\
\hline $\begin{array}{l}\text { Ecotoxicidad } \\
\text { acuática marina }\end{array}$ & kg 1,4-DB eq & $3.23 \mathrm{E}-04$ & $3.13 \mathrm{E}-04$ & $3.23 \mathrm{E}-04$ \\
\hline $\begin{array}{l}\text { Ecotoxicidad } \\
\text { terrestre }\end{array}$ & $\mathrm{kg} 1,4-\mathrm{DB}$ eq & $3.11 \mathrm{E}-06$ & 3.01E-06 & $3.11 \mathrm{E}-06$ \\
\hline $\begin{array}{l}\text { Oxidación } \\
\text { fotoquímica }\end{array}$ & $\mathrm{kg} \mathrm{C} 2 \mathrm{H} 4 \mathrm{eq}$ & 3.77E-06 & $3.66 \mathrm{E}-06$ & 3.77E-06 \\
\hline
\end{tabular}




\section{Tabla 4}

Resultados de la normalización para categorías de impacto (continuación)

\begin{tabular}{|c|c|c|c|c|}
\hline $\begin{array}{l}\text { Categoría de } \\
\text { impacto }\end{array}$ & Unidad & Demolición & Transporte & Reutilización \\
\hline Acidificación & $\mathrm{kg} \mathrm{SO} 2 \mathrm{eq}$ & $2.85 \mathrm{E}-05$ & $2.71 \mathrm{E}-06$ & $2.85 \mathrm{E}-05$ \\
\hline Eutrofización & kg PO4--- eq & 8.05E-06 & 7.34E-07 & $8.05 \mathrm{E}-06$ \\
\hline
\end{tabular}

Fuente: Elaboración propia a partir de datos de Simapro (PRÉ CONSULTANTS BV, 2018)

\section{Figura 6}

Resultados de la Normalización para Categorías de Impacto

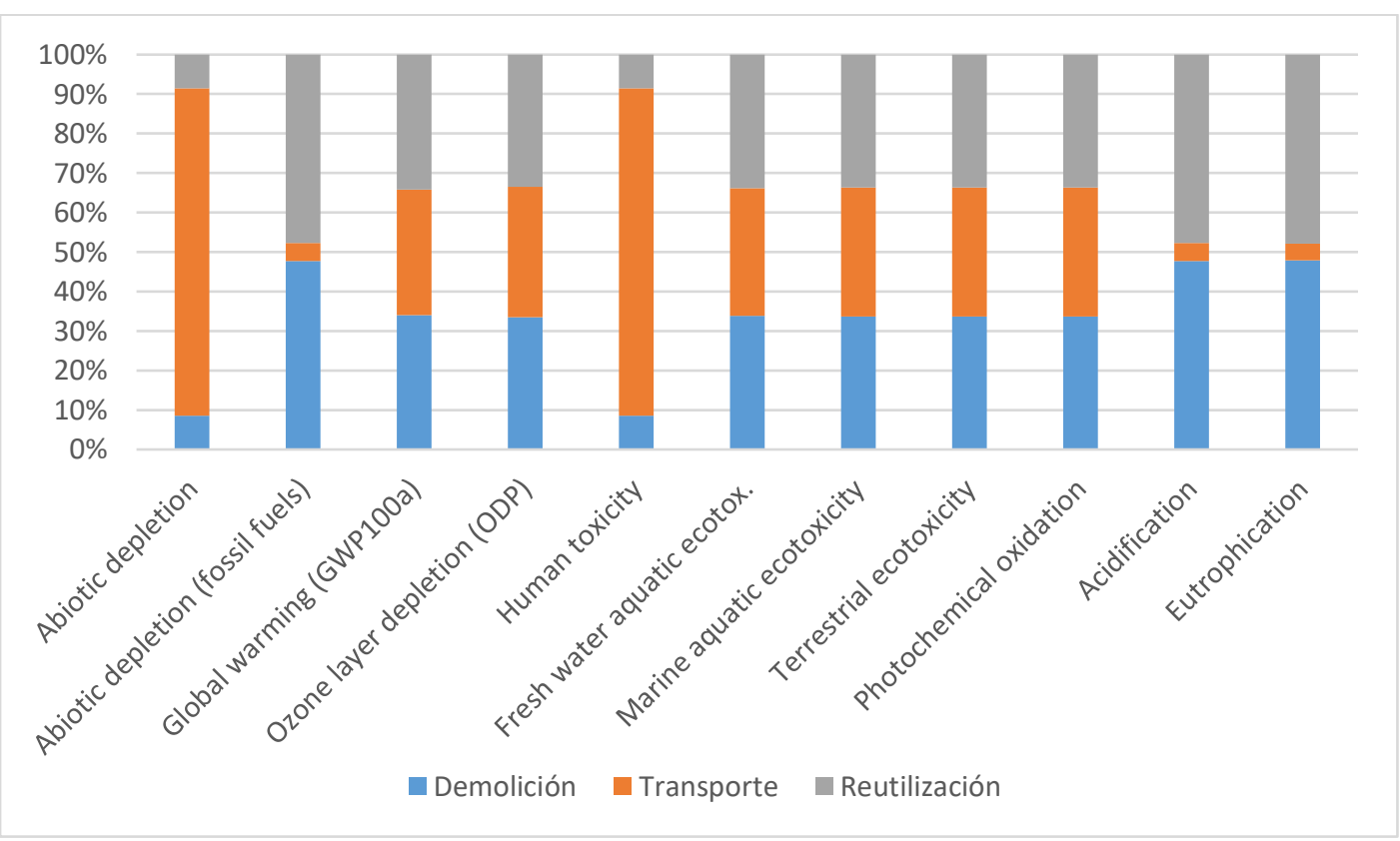

Fuente: Elaboración propia a partir de datos de Simapro (PRÉ CONSULTANTS BV, 2018)

Interpretación del ciclo de vida

De los resultados obtenidos en la evaluación del impacto del ciclo de vida, se concluye, que la mayor parte de las cargas contaminantes están generadas por la emisión de gases contaminantes producto de la combustión del diésel y combustibles reflejados en las categorías ambientales de agotamiento abiótico (combustibles fósiles), y calentamiento global.

\section{Conclusiones}

- Se investigó los posibles impactos ambientales en la reutilización del pavimento rígido en la vía Cuenca- Girón- Santa Isabel y las principales categorías de impacto ambiental que representan la mayor fuente de contaminación ambiental, son: Agotamiento abiótico (80\%), Calentamiento Global (30\%), Agotamiento abiótico (combustibles fósiles) (50\%), Toxicidad humana (80\%), Ecotoxicidad acuática marina (30\%), Ecotox acuático de agua dulce (30\%). 
- De igual manera, las categorías que contribuyen con menor impacto son: Agotamiento abiótico, Agotamiento de la capa de ozono (PAO), Ecotoxicidad terrestre, Oxidación fotoquímica, Acidificación, y Eutrofización, esto implica que, de las once categorías de impacto analizadas 5 categorías representan un mayor impacto, y mientras que 6 de las categorías contribuyen con un impacto menor, por lo que la reutilización del pavimento si genera menores impactos ambientales.

- Para la etapa de transporte se genera el mayor porcentaje de impactos ambientales, contribuyendo en más del $80 \%$ son el Agotamiento abiótico, y la Toxicidad humana, esto debido al consumo y producto de la combustión, mientras que las etapas de demolición y en la reutilización se han obtenido como el mayor impacto ambiental la categoría de Agotamiento abiótico (combustibles fósiles) con un valor casi del $50 \%$ y para el Calentamiento Global un valor de $30 \%$.

- El consumo de combustible y diésel es el que más incide en las 11 categorías de impacto ambientales analizadas, debido a que tiene gran influencia en todas las actividades por el uso de maquinaria pesada y el transporte de carga pesada de los materiales a la ubicación del proyecto.

- De acuerdo a los resultados obtenidos en la presente investigación se puede concluir que la reutilización del pavimento rígido si presenta menores impactos ambientales analizando todas las categorías de impacto planteadas, por lo que la metodología ACV puede ser utilizada como herramienta con ciertas limitaciones, para la evaluación ambiental y la factibilidad para su difusión en el país.

\section{Agradecimientos}

El presente artículo es parte del trabajo de investigación y titulación del Programa de Maestría en Construcción con Mención en Administración de la Construcción Sustentable de la Universidad Católica de Cuenca, por ello agradecemos a todos y cada uno de los instructores por los conocimientos e información brindados para la elaboración del trabajo.

\section{Referencias bibliográficas}

Ávila, F. (2020). Identificación de fallas geológicas en la vía Girón - Pasaje (Azuay). http://www.dspace.uce.edu.ec/bitstream/25000/21655/1/T-UCE-0012-FIG-024P.pdf

Baño Nieva, A., \& Escalera del Pozo, A. V. (2005). Guía de construcción sostenible. Instituto Sindical de Trabajo, Ambiente y Salud (ISTAS) ISTAS, 1-63.

Banco Interamericano de Desarrollo [BID]. (2014). El Cambio Climático y el BID: Creación de Resiliencia y Reducción de Emisiones. https://publications.iadb.org/bitstream/handle/11319/6692/CC_SpanishBRIK.pdf?s 
equence $=2 \&$ is Allowed $=\mathrm{y}$

Banco Interamericano de Desarrollo [BID]. (2018). Documento de Marco Sectorial de Cambio Climático. División de cambio climático.

Banco Interamericano de Desarrollo [BID]. (2019). Un vistazo al futuro: Diseño de la Red Vial Nacional de Resiliencia de Ecuador. Jean Pol Armijos Leray - Pablo Daza Donoso - T. Luke Young. https://blogs.iadb.org/transporte/es/un-vistazo-alfuturo-diseno-de-la-red-vial-nacional-de-resiliencia-de-ecuador/\#: :text=La ubicación geográfica del Ecuador y su geomorfología,como también\%2C incrementan la vulnerabilidad de las comunidades.

Camino Mogro, S., Bermudez Barrezuet, N., Chalen Vera, A., \& Romero Vallejo, D. (2018). Productividad en la Industria Ecuatoriana de la Construcción. Dirección Nacional de Investigación y Estudios de La Superintendencia de Compañias, Valores $y$ Seguros Del Ecuador, 1-30. file://C:/Users/DELL/Downloads/investigacion cientifica/Planteamiento del problema/Productividad_en_la_industria_ecuatoriana_de_la_construccion_20132017.pdf

Carlson, A. (2011). Studies made in Europe. January 2011.

Ecoinvent.org. (s.f.). What do the shortcuts, such as CH, RER, RoW and GLO mean? https://www.ecoinvent.org/support/faqs/methodology-of-ecoinvent-3/what-do-theshortcuts-such-as-ch-rer-row-and-glo-mean.html

Flintsch, G., \& Bryce, J. (2014). Sustainable pavement management. Green Energy and Technology, 204, 373-392. https://doi.org/10.1007/978-3-662-44719-2_13

García-Navarro, J., González-Díaz, M. J., Martínez, E., \& Redruello, I. (2012). Metodología para la evaluación de la sostenibilidad en autopistas: Cálculo del balance energético de la infraestructura. Informes de La Construccion, 64(528), 537-548. https://doi.org/10.3989/ic.11.156

Gobierno Provincial de Azuay. (2018). Plan de desarrollo y ordenamiento territorial del Azuay actualizado 2015 - 2030. Dk, 53(9), 1689-1699. http://app.sni.gob.ec/snilink/sni/PORTAL_SNI/data_sigad_plus/sigadplusdocumentofinal/0160000190001 _PDyOT_AZUAY 2015_17-08-2015_10-02-34.pdf

International Organization for Standardization [ISO]. (2004). Environmental Management - Life Cycle Assessment - Principles and Framework (ISO 14040:2006). Environmental Management System Requirements, 44(0).

MacK, J. W., Akbarian, M., Ulm, F. J., \& Louhghalam, A. (2017). Pavement-Vehicle Interaction Research at the MIT Concrete Sustainability Hub. Airfield and Highway Pavements 2017: Pavement Innovation and Sustainability - Proceedings of the International Conference on Highway Pavements and Airfield Technology 
2017, 2017-Augus, 160-173. https://doi.org/10.1061/9780784480946.015

Mendoza, J. F. (2014). Criterios de sustentabilidad para carreteras en méxico. 392.

Mercader, M. P., Marrero, M., Solís, J., Montes, M. V., \& Ramírez, A. (2010). Cuantificación de los recursos materiales consumidos en la ejecución de la cimentación. Informes de La Construccion, 62(517), 125-132. https://doi.org/10.3989/ic.09.000

Ministerio de Transporte y Obras Públicas del Ecuador. (2013). Norma Ecuatoriana Vial - NEVI 12. Norma Ecuatoriana Vial - NEVI 12, 1(00598), 83. http://www.obraspublicas.gob.ec/wp-content/uploads/downloads/2013/12/01-122013_Manual_NEVI-12_VOLUMEN_1.pdf

Perelli, M., \& Ruiz, L. P. (2017). Ciclo de vida ( CCV ) al caso particular de los firmes de carretera Approach from a Life Cycle Analysis and Life Cycle Cost Analysis. Revista Digital Del Cedex, 186(Ccv), 23-38.

PRÉ CONSULTANTS BV. (2018). PRÉ CONSUlTANTS BV. SIMAPRO. https://simapro.com/about/

Remache-Vinueza, B. (2017). Estudio de factibilidad para la implementación de la metodología de Análisis de Ciclo de Vida (ACV) en la valoración del impacto ambiental del sistema de transporte público de Tulcán. II Congreso Internacional de Ciencias Sociales y Económicas: Herramientas Para Fortalecer La Competitividad Sistémica de La Provincia Del Carchi, March, 1-13. https://www.researchgate.net/publication/323588967_Estudio_de_factibilidad_par a_la_implementacion_de_la_metodologia_de_Analisis_de_Ciclo_de_Vida_ACV_ en_la_valoracion_del_impacto_ambiental_del_sistema_de_transporte_publico_de_ Tulcan

Rivela Carvallal, B. (2012). Propuesto metodológica de aplicación sectorial de Análisis de Ciclo de Bida (ACV) para la evaluación ambiental de la edificación en España.

Stripple, H. (2001). Life Cycle Assessment of Road: A Pilot Study for Inventory Analysis. In IVL Swedish Environmental Research Institute.

Zapata, P., \& Gambatese, J. A. (2005). Energy Consumption of Asphalt and Reinforced Concrete Pavement Materials and Construction. Journal of Infrastructure Systems, 11(1), 9-20. https://doi.org/10.1061/(asce)1076-0342(2005)11:1(9)

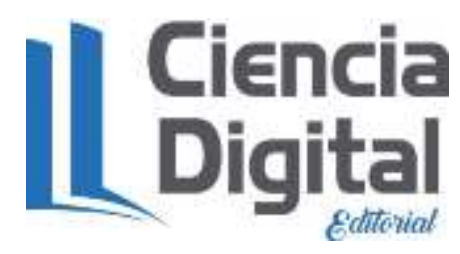




\section{PARA CITAR EL ARTÍCULO INDEXADO.}

Orellana Albán, S. T., \& Coronel Sacoto, D. F. (2021). Análisis del ciclo de vida aplicado para la evaluación ambiental en la reutilización del pavimento rígido. Caso de estudio vía Cuenca- Girón- Santa Isabel . ConcienciaDigital, 4(4.1), 131-151. https://doi.org/10.33262/concienciadigital.v4i4.1.1930

\section{Liencia}

El artículo que se publica es de exclusiva responsabilidad de los autores y no necesariamente reflejan el pensamiento de la Revista Conciencia Digital.

El artículo queda en propiedad de la revista y, por tanto, su publicación parcial y/o total en otro medio tiene que ser autorizado por el director de la Revista Conciencia Digital.

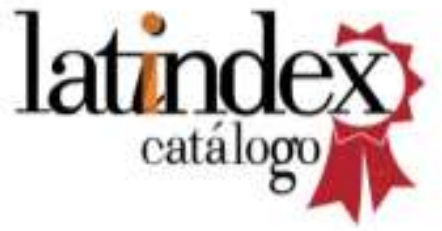

